\title{
Grand Unified Theories without the Desert
}

\author{
Alex Pomarol* \\ Theory Division, CERN, CH-1211 Geneva 23, Switzerland
}

(Received 5 June 2000)

\begin{abstract}
We present a grand unified theory (GUT) that has GUT fields with masses of the order of a TeV, but at the same time preserves (at the one-loop level) the success of gauge-coupling unification of the minimal supersymmetric standard model (MSSM) and the smallness of proton decay operators. This scenario is based on a five-dimensional theory with the extra dimension compactified as in the Randall-Sundrum model. The MSSM gauge sector and its GUT extension live in the 5D bulk, while the matter sector is localized on a $4 \mathrm{D}$ boundary.
\end{abstract}

PACS numbers: 11.10.Kk, 12.10.Dm, 12.60.Jv

1. Introduction.-One of the most fascinating challenges in particle physics is the unification of the forces of nature. Grand unified theories (GUTs) have been advocated as an extension of the standard model (SM) to unify the gauge interactions. Their immediate consequence is the explanation of charge quantization. The scale at which GUTs must replace the SM, however, must be very high, $M_{\mathrm{GUT}}>10^{15-16} \mathrm{GeV}$, in order to avoid higherdimensional operators that would lead to a large proton decay rate. Furthermore, a large $M_{\mathrm{GUT}}$ is also needed for gauge-coupling unification. Since the 4D gauge couplings evolve logarithmically with the energy scale, a large $M_{\mathrm{GUT}}$ is needed to allow the different gauge couplings of the SM to get closer and, eventually, unify. This is the case in the supersymmetric extension of the standard model [minimal supersymmetric standard model (MSSM)] where gauge couplings actually unify at $M_{\mathrm{GUT}} \sim 10^{16} \mathrm{GeV}$. Since supersymmetry is also needed for the stability of the weak scale versus the large GUT scale, supersymmetric GUTs provide a very appealing framework for physics beyond the SM. Nevertheless, we must face the fact that, since GUT fields will appear only at very high energies $M_{\mathrm{GUT}} \sim 10^{16} \mathrm{GeV}$, GUTs will never be tested in a direct way. These theories predict a big "desert" from the weak to the GUT scale.

Here we will present a GUT scenario without the desert. It has GUT fields of masses of the order of a TeV. This scenario has been proposed in Refs. [1,2] and is based on an extension of the MSSM to a five-dimensional theory with the extra dimension compactified as in the RandallSundrum model [3]. Differently from Ref. [3], however, we will consider the MSSM gauge sector and its GUT extension living in 5D with matter localized on a 4D boundary. In this Letter we will show that, even though the theory is five dimensional, gauge couplings get logarithmic corrections at the one-loop level. Therefore the theory predicts, as in the 4D MSSM case, the right values of the gauge couplings at low energies. We will also show that proton decay operators are suppressed by the high scale $M_{\mathrm{GUT}} \sim 10^{16} \mathrm{GeV}$.

Different attempts to obtain theories that, while predicting gauge-coupling unification as in the MSSM, do not have a desert between the weak and the GUT scale can be found in Ref. [4].

2. The setup.-Our setup is based on the RandallSundrum 5D model [3], where the bulk is a slice of $\mathrm{AdS}_{5}$. This corresponds to a 5D nonfactorizable geometry with the fifth dimension $y$ compactified on an orbifold, $S^{1} / \mathbb{Z}_{2}$, of radius $R$ with $0 \leq y \leq \pi R$. The orbifold fixed points at $y^{*}=0$ and $y^{*}=\pi R$ are 4D boundaries of the five-dimensional space-time. The metric is given by [3]

$$
d s^{2}=e^{-2 k y} \eta_{\mu \nu} d x^{\mu} d x^{\nu}+d y^{2},
$$

where $1 / k$ is the AdS curvature radius and $\eta_{\mu \nu}=$ $\operatorname{diag}(-1,1,1,1)$ with $\mu=1, \ldots, 4$. The fundamental scale in the 5D theory, $M_{5}$, is related with the 4D Planck mass, $M_{P}$, by $M_{5}^{3} \simeq k M_{P}^{2}$ (for $R>1 / k$ ). We assume that all the scales are of roughly the same order of magnitude $k \simeq M_{5} \simeq M_{P}$, with the radius of the extra dimension slightly larger, $R \sim 11 / k$. The effective scales on the two boundaries are very different. On the $y^{*}=0$ boundary the effective scale is $M_{P}$, while on the $y^{*}=\pi R$ boundary this is given by $k e^{-k \pi R} \sim \mathrm{TeV}$ (for the assumed value $R \sim 11 / k)$. We will hence call these two boundaries the $M_{P}$ boundary and the TeV boundary, respectively.

3. One-loop corrections to the gauge propagator at low energies. - Let us consider a 5D gauge boson $[1,5]$, $A_{M}(x, y)$, with $M=(\mu, 5)$, living in the slice of $\mathrm{AdS}_{5}$ described above. We will work in the gauge $A_{5}(x, y)=0$, so we only have to consider $A_{\mu}(x, y)$. At energies below the Kaluza-Klein (KK) masses, only the massless zero mode of the photon is relevant. This is given by $[1,5]$ $A_{\mu}(x, y)=\frac{1}{\sqrt{\pi R}} A_{\mu}^{(0)}(x)+\cdots$. This corresponds to a $4 \mathrm{D}$ massless state with a $y$-independent wave function. We want to calculate the one-loop corrections to the propagator of this massless gauge boson. For simplicity, we will consider a 5D scalar QED theory, and calculate the corrections generated by a 5D scalar $\phi$ with charge 1 and even under the $\mathbb{Z}_{2}$. We will regularize this theory with a $5 \mathrm{D}$ Pauli-Villars (PV) field $\Phi$ of mass $\Lambda$. This mass corresponds to the cutoff of the theory that we will take to be $\Lambda \lesssim k$. Let us decompose the 5D scalar fields $\phi$ and $\Phi$ in KK modes. This has been done in Refs. [2,6]. For a 
5D scalar particle of mass $M$, the approximate KK mass spectrum for $M<k$ is given in Table I.

We have defined the $n=0$ mode as the mode that becomes massless in the limit $M \rightarrow 0$. For $M$ of order $k$, this mode becomes heavier than some of the KK states since $m_{\mathrm{KK}}=\pi k e^{-k \pi R} \ll k$ for $R>1 / k$. This is very different from compactifications in a slice of flat space where the $n=0$ mode (defined as explained above) is always the lightest state. For the scalar $\phi$ and the PV field $\Phi$ we can obtain the KK spectrum using Table I with $M=0$ and $M=\Lambda$, respectively. From this KK decomposition we can already infer the magnitude of the quantum corrections. For each KK mode of the field $\phi$ there is a KK mode of the PV field whose mass acts as a cutoff scale. Since the masses of the KK modes of $\phi$ and $\Phi$ are of the same order of magnitude, we do not expect large corrections from them. Nevertheless, the zero mode of the PV field is very heavy, $\Lambda / \sqrt{2}$, in contrast with the zero mode of $\phi$ that is massless. Therefore we expect a large correction coming from this large mass splitting of the zero modes that will reproduce the quantum corrections of an ordinary 4D theory.

To see this explicitly, let us now calculate the one-loop contribution from the scalar $\phi$ to the propagator of the photon $A_{\mu}^{(0)}$. Defining the photon self-energy by $\Pi_{\mu \nu}(q)=$ $\left[q^{2} \eta_{\mu \nu}-q_{\mu} q_{\nu}\right] \Pi(q)$, we find, at zero momentum, that the one-loop contribution is given by

$$
\Pi(0)=\frac{b_{0}}{8 \pi^{2}} \ln \frac{m_{0}^{\phi}}{m_{0}^{\Phi}}+\frac{b_{\mathrm{KK}}}{8 \pi^{2}} \sum_{n=1}^{\infty} \ln \frac{m_{n}^{\phi}}{m_{n}^{\Phi}},
$$

where $m_{0}^{\phi}$ and $m_{n}^{\phi}$ are, respectively, the masses of the zero mode and $n$-KK mode of the field $\phi$, and similarly for $\Phi$. We denote by $b_{0}$ and $b_{\mathrm{KK}}$ the beta-function coefficients of the zero mode and KK modes, respectively. In the example here we have $b_{0}=b_{\mathrm{KK}}=1 / 3$. Using Table I with $M=0$ and $M=\Lambda$, respectively, for $\phi$ and the PV field $\Phi$, we obtain

$$
\begin{aligned}
\Pi(0) \simeq & \frac{b_{0}}{8 \pi^{2}} \ln \frac{\mu}{\Lambda}+\frac{b_{\mathrm{KK}}}{8 \pi^{2}} \\
& \times \int_{1}^{e^{k \pi R}} d n \ln \left(\frac{n+1 / 4}{n+1 / 4+\Lambda^{2} /\left(8 k^{2}\right)}\right),
\end{aligned}
$$

where as usual we have introduced an infrared cutoff $\mu$, and we have replaced the sum over $n$ by an integral. Evaluating the integral and considering that $\Lambda \lesssim k$, we

TABLE I. Approximate KK mass spectrum of a 5D scalar of mass $M$. We have defined $\alpha=\sqrt{4+\left(M^{2} / k^{2}\right)}$ and $m_{\mathrm{KK}}=$ $\pi k e^{-k \pi R}$. We have neglected corrections to the spectrum of $\mathcal{O}(1 / n)$. KK modes with masses close to $M$ or $k$ can have deviations of $\mathcal{O}(1)$ in their masses from the values given here. Since these deviations only affect a few KK, they will be neglected.

\begin{tabular}{lccc}
\hline \hline & $n=0$ & $n=1$ to $n \lesssim e^{k \pi R}$ & $n \gtrsim e^{k \pi R}$ \\
\hline KK masses & $M / \sqrt{2}$ & $\left(n+\frac{\alpha}{2}-\frac{3}{4}\right) m_{\mathrm{KK}}$ & $n m_{\mathrm{KK}}$ \\
\hline \hline
\end{tabular}

obtain

$$
\Pi(0) \simeq \frac{b_{0}}{8 \pi^{2}} \ln \frac{\mu}{\Lambda}+\frac{b_{\mathrm{KK}}}{64 \pi^{2}} \frac{\Lambda^{2}}{k^{2}} \ln \frac{5 m_{\mathrm{KK}}}{4 \pi k} .
$$

For $\mu \ll \Lambda<k$ the $\mathrm{KK}$ contribution is small and can be neglected. We then obtain that the contribution to the gauge boson propagator is dominated by the zero mode and gives exactly the same contribution as in four dimensions:

$$
\Pi(0) \simeq \frac{b_{0}}{8 \pi^{2}} \ln \frac{\mu}{\Lambda} .
$$

It is important to see if the result of Eq. (5) can be understood as a running of the gauge coupling similar to the 4D case. Of course, this cannot be the case if matter is localized on the $\mathrm{TeV}$ boundary, since on that boundary our effective scale (cutoff scale) is $\mathrm{TeV}$, and above this energy effects must be considered from the fundamental (string) theory. Nevertheless, if matter is localized on the $M_{P}$ boundary, where the effective scale is $M_{P}$, we will show in the next section that the effective gauge couplings can be considered to run logarithmically with the energy similarly to the $4 \mathrm{D}$ case.

4. The 5D gauge propagator at high energies.-In order to understand what is the behavior of the theory at energies above the $\mathrm{TeV}$, we will derive here the $5 \mathrm{D}$ propagator of the gauge boson. Since we are interested in the propagator at high energies from the point of view of the $M_{P}$ boundary, we will consider the limit $R \rightarrow \infty$.

Let us take the gauge $A_{5}(x, y)=0$ and consider only the transverse part of $A_{\mu}(x, y)$, i.e., we impose $\partial^{\mu} A_{\mu}(x, y)=0$. It is shown in Ref. [7] that the transverse part decouples from the nontransverse part in the equations of motion. Moreover, only the propagator of the transverse part is relevant to sources localized on the $M_{P}$ boundary since the current there is transverse, $\partial^{\mu} J_{\mu}=0$. Following similar steps to those in the graviton case $[8,9]$, we want to calculate the Green function for the gauge boson defined as

$$
A_{\mu}(x, y)=\int d^{4} x^{\prime} d y^{\prime} \sqrt{-g} G\left(x, y ; x^{\prime}, y^{\prime}\right) e^{2 k y^{\prime}} J_{\mu}\left(x^{\prime}, y^{\prime}\right),
$$

with $\partial^{\mu} J_{\mu}=0$. It can be shown that $e^{k\left(y+y^{\prime}\right)} G\left(x, y ; x^{\prime}, y^{\prime}\right)$ is also the Green function of a scalar with mass $-3 k^{2}+$ $2 k \delta(y)$ [2]. Let us change the extra dimensional coordinate to $z=e^{k y} / k$. Taking the 4D Fourier transform of the Green function

$$
G\left(x, z ; x^{\prime}, z^{\prime}\right)=\int \frac{d^{4} p}{(2 \pi)^{4}} e^{i p\left(x-x^{\prime}\right)} G_{p}\left(z, z^{\prime}\right),
$$

we have that $G_{p}\left(z, z^{\prime}\right)$ must satisfy the equation

$$
\left[\partial_{z}^{2}-\frac{1}{z} \partial_{z}-p^{2}\right] G_{p}\left(z, z^{\prime}\right)=z k \delta\left(z-z^{\prime}\right) .
$$


Solving Eq. (8) with the Neumann boundary condition on the $M_{P}$ boundary, we find

$$
G\left(x, z ; x^{\prime}, z^{\prime}\right)=\frac{i \pi k}{2} z z^{\prime} \int \frac{d^{4} p}{(2 \pi)^{4}} e^{i p\left(x-x^{\prime}\right)}\left[\frac{J_{0}(i p / k)}{H_{0}^{(1)}(i p / k)} H_{1}^{(1)}(i p z) H_{1}^{(1)}\left(i p z^{\prime}\right)-J_{1}\left(i p z_{<}\right) H_{1}^{(1)}\left(i p z_{>}\right)\right],
$$

where $H_{\nu}^{(1)}=J_{\nu}+i Y_{\nu}$ is the Hankel function of order $\nu$, $J_{\nu}$ and $Y_{\nu}$ are Bessel functions, and we have defined $z_{>}$ $(z<)$ as the greater (lesser) of $z$ and $z^{\prime}$. In the case where the coordinate $z^{\prime}$ is on the $M_{P}$ boundary, $z^{\prime}=1 / k$, the Green function simplifies to

$$
G\left(x, z ; x^{\prime}, \frac{1}{k}\right)=-i k z \int \frac{d^{4} p}{(2 \pi)^{4}} e^{i p\left(x-x^{\prime}\right)} \frac{1}{p} \frac{H_{1}^{(1)}(i p z)}{H_{0}^{(1)}(i p / k)} .
$$

We are interested in the limit $r=\left|x-x^{\prime}\right| \gg 1 / k$ where the Green function is dominated by the small-momentum values of the Fourier transform $(p \ll k)$ and the Hankel function $H_{0}^{(1)}(i p / k)$ is approximately $H_{0}^{(1)}(i p / k)=$ $i \frac{2}{\pi}\left[\ln \frac{p}{2 k}+\gamma\right]+\mathcal{O}\left(\frac{p^{2}}{k^{2}}\right)$.

Let us now study the Green function of Eq. (10) in two different limits. First let us consider the propagator at large $z, z \gg r$. In this case we find a falloff of the Green function

$$
G\left(x, z \gg r ; x^{\prime}, \frac{1}{k}\right) \sim \frac{k}{z^{2} \ln (k z)}
$$

that is similar to the graviton case [8,9]. Equation (11) implies that at large momentum $p \gtrsim 1 / z=k e^{-k y}$ the $M_{P}$ boundary decouples from the TeV boundary. Let us now consider the opposite limit, $r \gg z$, that also corresponds to the case when $z=1 / k$, i.e., the gauge propagator on the $M_{P}$ boundary. In this case we have

$$
G\left(x, \frac{1}{k} ; x^{\prime}, \frac{1}{k}\right) \simeq k \int \frac{d^{4} p}{(2 \pi)^{4}} e^{i p\left(x-x^{\prime}\right)} \frac{1}{p^{2} \ln (p / k)} .
$$

From Eq. (12) we can derive the static potential on the $M_{P}$ boundary:

$$
V(r) \sim \frac{k}{r} \frac{1}{\ln (k r)} .
$$

We see that it differs from the Coulomb potential in $4 \mathrm{D}$ by a logarithmic term. It means that the gauge coupling on the $M_{P}$ boundary grows, at the tree level, logarithmically with the energy. This is in contrast with $5 \mathrm{D}$ theories in flat space where at the classical level the coupling grows linearly with the energy. In a theory with finite $R$ this "running" will be present at energies above the mass of the first KK mode $\sim m_{\mathrm{KK}}=\pi k e^{-k \pi R}$ (below $m_{\mathrm{KK}}$ we have a single massless gauge boson as in $4 \mathrm{D}$ ). We then have

$$
g^{2}\left(p>m_{\mathrm{KK}}\right) \simeq g^{2}\left(p \sim m_{\mathrm{KK}}\right) \frac{\pi k R}{\ln (k / p)} .
$$

This mild logarithmic evolution of the gauge coupling allows us to go to high energies without entering in the strong coupling regime. We must stress that this running of the gauge coupling is a tree-level effect, not a quantum one. As a consequence, it will be universal for the differ- ent groups of the SM and will not affect gauge-coupling unification.

From the tree-level behavior of the propagator in Eq. (9), we learn that the theory remains weakly coupled for $p \lesssim$ $1 / z$. This suggests that the theory can be renormalized as long as we keep our cutoff scale below $1 / z$, i.e., $\Lambda \lesssim$ $k e^{-k y}$. Notice that this cutoff scale depends on the position in the extra dimension. This should be expected in a theory with the metric (1), since the effective scale of the 4D space-time at the position $y$ is given by $\sim k e^{-k y}$. Using the cutoff $\Lambda=k e^{-k y}$, we can calculate quantum corrections in a very simple way. We just need the $5 \mathrm{D}$ propagators for $r>z$. For the case of the 5D massless scalar discussed in the previous section, the propagator behaves as in $4 \mathrm{D}[8,9]$, $k \int d^{4} p /(2 \pi)^{4}\left(1 / p^{2}\right)$, giving then the same one-loop correction to the gauge coupling as in $4 \mathrm{D}$.

5. GUTs in a slice of $A d S_{5}$. - Let us now proceed to show that theories with gauge bosons in a slice of $\mathrm{AdS}_{5}$ can have gauge-coupling unification. We will take a top-down approach. We will assume that we have a supersymmetric GUT in the slice of $\mathrm{AdS}_{5}$ and show that this theory, when broken to the MSSM group, leads to a successful prediction for the gauge couplings at low energies.

As a toy example, let us consider an $\mathrm{SU}(5)$ theory. Because of the $\mathbb{Z}_{2}$ orbifold symmetry, the massless gauge sector of this theory consists of a $N=1$ vector multiplet [2]. They contain the SM gauge bosons plus the GUT gauge bosons, $X$ and $Y$, that complete the $\mathrm{SU}(5)$ representation. The KK spectrum consists of $N=2$ vector multiplets with masses $\sim\left(n-\frac{1}{4}\right) m_{\mathrm{KK}}$. Let us now consider that on the $M_{P}$ boundary we have a chiral supermultiplet, in the 24 representation of $\mathrm{SU}(5)$, whose scalar gets a vacuum expectation value (VEV) equal to $M_{\mathrm{GUT}} \sim$ $10^{16} \mathrm{GeV}$ (slightly below $k \simeq M_{P}$ ) breaking the SU(5) group down to the MSSM. This can be achieved in the same way as in ordinary 4D SU(5) theories, since our theory on the boundary is $4 \mathrm{D} N=1$ supersymmetric. It is easy to calculate the KK spectrum of the resulting theory. The $n=0$ MSSM gauge bosons remain massless, while the GUT gauge bosons, $X$ and $Y$, have masses $M_{X, Y} \simeq M_{\mathrm{GUT}}$. (More precisely, we find that $M_{X, Y}$ is determined by the equation $M_{X, Y}\left[\ln \left(M_{X, Y} / 2 k\right)+\right.$ $\gamma+1 / 2]+M_{\mathrm{GUT}}^{2} / M_{X, Y}=0$.) The KK mass spectrum $(n \geq 1)$, however, is not modified by the VEV of the 24 [up to corrections of $\mathcal{O}\left(M_{n}^{2} / k^{2}\right)$ ] and therefore the KK modes approximately respect the SU(5) symmetry. Consequently, only the zero modes (as we claimed before) will give a relative one-loop contribution to the SM gauge couplings which, at energies $\mu$, is given by

$$
\frac{1}{\alpha_{i}}=\frac{1}{\alpha_{j}}+\frac{b_{i}-b_{j}}{2 \pi} \ln \frac{M_{\mathrm{GUT}}}{\mu},
$$


where $b_{i}$ is the contribution of the massless modes to the beta-function coefficient of the MSSM gauge group $i$. Therefore, in order to have the same predictions for the gauge coupling as in 4D supersymmetric GUTs, we must demand that the massless states of the theory be those of the MSSM. This will be the case of the gauge sector, as we already explained. For the Higgs sector we can, as usual, assume that they arise from a $\mathbf{5}$ and $\overline{\mathbf{5}}$ of $\mathrm{SU}(5)$. Since we need to have only the $\mathrm{SU}(2)_{L}$-doublet light, we will need a mechanism that provides a doublet-triplet mass splitting inside the $\mathbf{5}$ and $\overline{\mathbf{5}}$. Several mechanisms exist in the literature for $4 \mathrm{D}$. It is not clear if these mechanisms can also work in 5D. Nevertheless, we can rely on these mechanisms by assuming that the Higgs live on the $M_{P}$ boundary.

Finally, we must implement the matter sector. Since they form complete SU(5) multiplets, $\overline{\mathbf{5}}$ and 10, they are irrelevant to gauge-coupling unification (they will not contribute at the one-loop level to the relative corrections to the gauge couplings). The matter sector, however, must satisfy important constraints from proton decay. Since there are very light KK modes of the $X$ and $Y$ bosons $\left(m_{\mathrm{KK}} \sim \mathrm{TeV}\right)$, we must worry about proton decay operators induced by these modes. If we analyze the $y$-dependent wave function of these modes, however, we find that they are peaked on the $\mathrm{TeV}$ boundary [1]. Therefore, proton decay constraints can be satisfied by just placing the matter sector on the $M_{P}$ boundary. In this case, even if we sum over the full KK tower of the $X$ and $Y$ bosons, we obtain that the strength of the dimension-six proton decay operator is given by

$$
\sum_{n} g_{n}^{2} \frac{1}{m_{n}^{2}} \simeq g^{2} \frac{\pi k R}{M_{\mathrm{GUT}}^{4}} \int_{0}^{M_{\mathrm{GUT}}} m d m=g^{2} \frac{\pi k R}{2 M_{\mathrm{GUT}}^{2}}
$$

where $g_{n}$ and $m_{n}$ are, respectively, the coupling to the $M_{P}$ boundary and the mass of the $\mathrm{KK}$ of the $X, Y$ bosons that can be derived following Ref. [2]. We see that the result is similar to that in a 4D theory where one finds $g^{2} / M_{\text {GUT }}^{2}$. The operator (16) is, however, slightly larger in our theory than in $4 \mathrm{D}$ theories because of the factor $\pi k R$ in Eq. (16). This enhancement is due to the fact that the gauge coupling grows (at tree level) with the energy according to Eq. (14). Notice that, at the scale $M_{\mathrm{GUT}}$, the theory is close to the strong coupling regime. This is why we expect in these GUTs a proton decay rate for $p \rightarrow \pi e$ closer to the experimental limit than in 4D GUTs.

Up to now we have just assumed that $m_{\mathrm{KK}}$ (approximately the mass of the lightest KK state) is an independent parameter of the theory that we have taken to be close to the weak scale by choosing $R \simeq 11 / k$. Nevertheless, it would be interesting to relate $m_{\mathrm{KK}}$ with the weak scale. One way to do this is by associating the supersymmetry-breaking scale with $m_{\mathrm{KK}}$. A realization of this is given in Ref. [2]. By assuming different boundary conditions for bosons and fermions on the $\mathrm{TeV}$ boundary, we can get a fermion- boson mass splitting of $\mathcal{O}\left(m_{\mathrm{KK}}\right)$. This breaks supersymmetry and induces a Higgs mass of $\mathcal{O}\left(m_{\mathrm{KK}}\right)$ [10]. If this mass is negative, this will trigger electroweak symmetry breaking. This scenario therefore links the scale $m_{\mathrm{KK}}$ with the weak scale.

Although this theory resembles the ordinary 4D supersymmetric GUT, it has very different implications at $\mathrm{TeV}$ energies. While 4D supersymmetric GUTs predict that only the MSSM fields have masses of the order of the weak scale, with a big desert up to the GUT scale, our theory has plenty of new physics at the TeV. There are the KK states not only of the SM but also of the GUT fields and graviton. It has been shown in Ref. [1] that the KK modes of the SM gauge bosons have sizable couplings to the SM fermions living on the $M_{P}$ boundary $(\simeq 0.2 g)$ and therefore they could be seen as resonances in $\mathrm{TeV}$ colliders. On the other hand, the KK modes of the GUT fields couple very weakly to the $M_{P}$ boundary (this is why the proton decay rate is small). These modes, however, can be produced at $\mathrm{TeV}$ energies by processes mediated by virtual SM gauge bosons (that live in the 5D bulk and propagate between the two boundaries). At these energies, also graviton KK modes can be produced. In fact, since the effective scale on the $\mathrm{TeV}$ boundary is $\sim k e^{-k \pi R} \sim \mathrm{TeV}$, quantum gravity or string effects can be important and possible to test.

We thank Jaume Garriga, Tony Gherghetta, and Riccardo Rattazzi for very useful discussions. This work has been supported in part by the Spanish CICYT Contract No. AEN99-0766.

* On leave of absence from IFAE, Universitat Autònoma de Barcelona, E-08193 Bellaterra, Barcelona.

[1] A. Pomarol, Phys. Lett. B 486, 153 (2000).

[2] T. Gherghetta and A. Pomarol, Nucl. Phys. B586, 141 (2000).

[3] L. Randall and R. Sundrum, Phys. Rev. Lett. 83, 3370 (1999).

[4] I. Antoniadis, Phys. Lett. B 246, 377 (1990); K. R. Dienes, E. Dudas, and T. Gherghetta, Phys. Lett. B 436, 55 (1998); Z. Kakushadze, Nucl. Phys. B548, 205 (1999); I. Antoniadis and C. Bachas, Phys. Lett. B 450, 83 (1999); N. Arkani-Hamed, S. Dimopoulos, and J. March-Russell, hep-th/9908146.

[5] H. Davoudiasl, J. L. Hewett, and T. G. Rizzo, hep-ph/ 9911262.

[6] W. D. Goldberger and M. B. Wise, Phys. Rev. D 60, 107505 (1999); S. Chang, J. Hisano, H. Nakano, N. Okada, and M. Yamaguchi, hep-ph/9912498.

[7] H. Liu and A. A. Tseytlin, Phys. Rev. D 59, 086002 (1999).

[8] J. Garriga and T. Tanaka, Phys. Rev. Lett. 84, 2778 (2000).

[9] S. B. Giddings, E. Katz, and L. Randall, J. High Energy Phys. 0003, 023 (2000).

[10] See J. Garriga, O. Pujolas, and T. Tanaka, hep-th/0004109, for a formalism to calculate scalar masses in this background. For a flat space, see A. Delgado, A. Pomarol, and M. Quiros, Phys. Rev. D 60, 095008 (1999). 